\title{
Research on Intelligent Agricultural Planting System Based on Internet of Things Technology
}

\author{
Yunsheng Chen, Shuduo Zhao, Yunxu Zhou \\ Southwest Petroleum University, Chengdu, China \\ Email: yunshengchen@163.com
}

How to cite this paper: Chen, Y.S., Zhao, S.D. and Zhou, Y.X. (2018) Research on Intelligent Agricultural Planting System Based on Internet of Things Technology. Journal of Computer and Communications, 6, 54-60.

https://doi.org/10.4236/jcc.2018.66005

Received: May 28, 2018

Accepted: June 24, 2018

Published: June 27, 2018

Copyright $\odot 2018$ by authors and Scientific Research Publishing Inc. This work is licensed under the Creative Commons Attribution International License (CC BY 4.0).

http://creativecommons.org/licenses/by/4.0/

\section{Open Access}

\begin{abstract}
Agriculture is the basic industry that concerns the national economy and people's livelihood. In the process of transforming to modern agriculture, the traditional agriculture in our country faces the problems of ensuring the quality of agricultural production, adjusting agricultural industrial structures, improving the low production efficiency and low utilization rate of resources, and environmental pollution, thus it cannot meet the needs of sustainable agricultural development. Therefore, the research on intelligent agriculture technology is imperative. This paper analyzes the key technologies of Internet of things applied in the intelligent agriculture, presents the application of Internet of things technology in agricultural planting system, constructs the intelligent agricultural planting system based on the Internet of things technology, and designs the framework of the management platform.
\end{abstract}

\section{Keywords}

Internet of Things, Intelligent Agriculture, Sensor, Wireless Transmission, Data Collection

\section{Introduction}

The Internet of things (IOT) is the Internet for connecting things. At present, the more accurate definition of the Internet of things is that it is an information network, connecting things and things, people and things, people and people, through various perception devices and systems, bar codes and QR codes, and global positioning systems in accordance with agreed communication protocols, in order to realize intelligent identification, positioning, tracking, monitoring and management through information exchanges by various access networks, Internet and other networks. In this network, every object can be addressed and controlled, and every object can communicate. This has two meanings. First, the 
core and foundation of the Internet of things is still the Internet, and it is an extended and expanded network based on the Internet. Second, its user sides extend to any object for information exchanges and communications while intelligence system is a computer system that can produce human intelligent behaviors.

With the rapid development of China's economy in the past 30 years, the shortage of agricultural production resources and the excessive consumption of agricultural resources, the restriction on agricultural development becomes more and more obvious. Agricultural Internet of things (IoT) applies advanced IoT technologies such as sensing, communication and data processing to the agricultural field. Constructing intelligent agriculture system is an effective way to solve the problem of lagging agricultural development. The Internet of things (IoT) is a network for the purpose of perception, which realizes the comprehensive interconnection between people and people, people and things, things and things. The Internet of things and intelligent system have achieved huge successes in various fields, such as biometric identification [1], wireless communication [2], systems biology [3], etc., among which the intelligent agriculture is also one of them.

\section{Internet of Things Technology and Intelligent Agriculture}

\subsection{The Background of Intelligent Agriculture Coming into Being}

Agricultural information technology is an important part of modern agricultural science and technology in China. It is the development direction of China's modern agriculture to vigorously promote the integration of information technology and agricultural modernization. "Intelligent agriculture" refers to an agricultural system that uses the Internet of things technology, monitor real-time agricultural planting environments [4] through the corresponding intelligent sensors, and convey the corresponding data collected by data acquisition equipment to the control center by wireless network system, then adjusts the agricultural planting environment, and intelligently control the needed environments for crops' healthy growth, such as temperature, humidity, lighting, soil temperature, water content, and timely irrigation system, in order to realize automatic detection of comprehensive ecological information of agricultural planting, automatic control and intelligent management of the environment.

\subsection{The Main Applications of Internet of Things Technology in Intelligent Agriculture}

\subsubsection{Internet of Things Technology Realizes the Intelligent Management of Agricultural Planting System}

By installing intelligent sensor equipment and other intelligent control systems in the agricultural seed system, we can realize the real-time monitoring of various parameters in the planting environment of crops, timely grasp some parameters of the growing environment of crops, and timely control such as irrigation system and temperature and humidity control systems according to the 
changes of parameters. By collecting, analyzing and controlling air temperature and humidity, soil temperature and humidity, light intensity and $\mathrm{CO}_{2}$ content, we can control the best growing environment of crops. Applying the biological information acquisition method to wireless sensor nodes can provide scientific basis for the precise control of greenhouse [5].

\subsubsection{Internet of Things Technology Achieves the Effective Supervisions of Quality and Safety of Agricultural Products}

At present, the most serious problem that the China's agricultural products market faces is the quality and safety of agricultural products. The toxic food incidents have been numerous. The root of this problem lies in the extensive management of agricultural products in China. In the process of producing, circulation and products trading, the market supervision is not well regulated. The current agricultural Internet of things technology can solve this problem well. It widely uses the key technologies, such as electronic identification, barcode, sensor network, Internet of things middleware and network platform technology to achieve transparency and real-time monitoring of agricultural products information from producing, storage, transportation and trading. Thus, the whole process of agricultural products from farmland to table can be managed and controlled, and the quality of agricultural products can be safely and effectively regulated [6].

\subsubsection{Agricultural Intelligent Detection and Cultivation System}

The structure of agricultural intelligent monitoring and cultivation system is composed of wireless network, cultivating control equipment, gateway and control center. The wireless communication network consists of several cluster networks deployed in the monitoring area. The wireless communication technology node is responsible for monitoring temperature, humidity, light intensity and other agricultural information, collecting the information and transmitting it to the gateway through the wireless communication network. Different kinds of agricultural information have different effects on crops. Light, for example, is essential for photosynthesis in crops. So we place a light intensity sensor above the crops to monitor the environmental light intensity in real time, so as to grasp the light intensity of the growing environment of crops in time. The environmental temperature directly affects the growth rate and development of crops, and air humidity is also an important factor affecting crop's growth and development. So an air humidity sensor should be put around the crops. The wireless communication network is connected to the transmission network through a gateway with adaptive switching function, and the data is transmitted to the control center. The control center processes the received data and saves it to the database, summarizes and analyzes the collected information, issues feedback control instructions with combination of expert decision system, timely and accurately identify and solve problems to guide agricultural production. By means of Internet, producers and technical researchers can monitor the collected agricultural information at any time and any place, and track the growth of crops in 
real time. The technical personnel in charge of crop production will formulate reasonable breeding strategies according to the growing conditions and actual needs of the crops [7].

\subsubsection{Agricultural Products Transportation Management and Control System}

For agricultural products transportation management and control system, the control orders are issued by remote control center. After the vehicle leaves the management center, the self-designed DTU (date transfer unit) installed on the vehicle will feed the current vehicle location information back to the control center.

Through the DTU unit on the transport vehicle, the current longitude and latitude, speed, altitude and satellite timing of the vehicle are sent to the remote control center by wireless remote transmission. The control center then establishes the function correspondence relation between the GPS data which is sent remotely and the electronic map, and regards the transport vehicle as a moving point on the map. In this way, the traffic route, speed and condition of the point can be monitored transparently and unerringly, and thus, the intelligent control and management can be realized. The information such as the temperature and humidity of agricultural products in transportation can be monitored by sensors in the cabinet loading agricultural products. The control center has instant voice communication platform composed of GSM and GPRS, and it can get in touch with the working transport vehicles through the central computer control software, which is of great significance to eliminate emergency and practical problems.

\section{Intelligent Agricultural Planting System Design}

\subsection{Principle of Intelligent Agricultural Planting System}

The Internet of things technology is applied to the control of intelligent agricultural planting system, and the principles are as follows: Install the intelligent sensors and associated intelligent control systems in planting areas where crops are concentrated, and intelligently control the air, temperature and water needed for crop growth by the collected data analyzed by computers, thus realize the precision agriculture.

Sensors in the intelligent agricultural planting system realize real-time monitoring of planting environment. It collects the relevant information, determines the problems in the planting environment, starts the corresponding irrigation system, thermal insulation system and supplementary lighting system in real time in order to ensure that the crops have a suitable growing environment. So as to realize the automation and intelligence level of modern agriculture, reduce the share of resources, and improve the production efficiency and product quality of agricultural products [8].

\subsection{Construction of Intelligent Agricultural Planting System}

Intelligent agricultural planting system based on Internet of things technology 
consists of sensor, wireless collector, wireless controller, wireless coordinator and environment monitor. It is shown in Figure 1.

Intelligent agricultural planting system based on Internet of things includes the following parts:

1) Planting environment monitoring: Use corresponding sensors to realize real-time monitoring of soil moisture, air temperature and humidity, lighting and automatic irrigation system in planting environment.

2) Data collection system: Use ZigBee wireless transmission mode to upload data, which is monitored by sensors in real time. The ZigBee sending module transmits sensor values to the ZigBee coordinator node. Sampling ZigBee wireless communication mode has the advantages of flexible deployment and convenient expansion.

3) Network transmission system: Using wireless transmission system, this system will collect the data, and transmit it to the server through wireless network. The data platform system is responsible for storing the collected data, processing information and issuing control instructions. It provides users with analysis and decision-making basis, so that users can query through computers, mobile phones and other terminals at any time and any place.

4) Information systems control: The system consists of control node, relay extension module and corresponding electromechanical actuator. It can freely control various agricultural production equipment by relay.

\subsection{Construction of Intelligent Agricultural Planting System Management Platform}

The management platform of intelligent agricultural planting system is shown in Figure 2. It is divided into five parts: sensor information acquisition, automatic monitoring, manual intervention, intelligent analysis and remote control.

For example, Guangxi province has many grape planting enterprises, and Nanning Xiangsi grape agricultural technology co., LTD is one of the outstanding enterprises. Xiangsi grape takes "technology first" as its philosophy. It actively studies and exchanges with universities and laboratories all over the country, and officially launched "intelligent agricultural monitoring system" in various parks in 2012.

It makes full use of "Internet of things, cloud computing, mobile Internet" and other technologies to upgrade the traditional grape planting technology, as a result, it not only reduces manpower cost, but also ensures grape quality through automatic remote control.

\section{Conclusion}

Agriculture is the basic industry that concerns the national economy and people's livelihood in China. And the restriction on agricultural development becomes more and more obvious due to the shortage of agricultural production resources and the excessive consumption of agricultural resources. The research on intelligent agricultural planting system based on Internet of things technology 


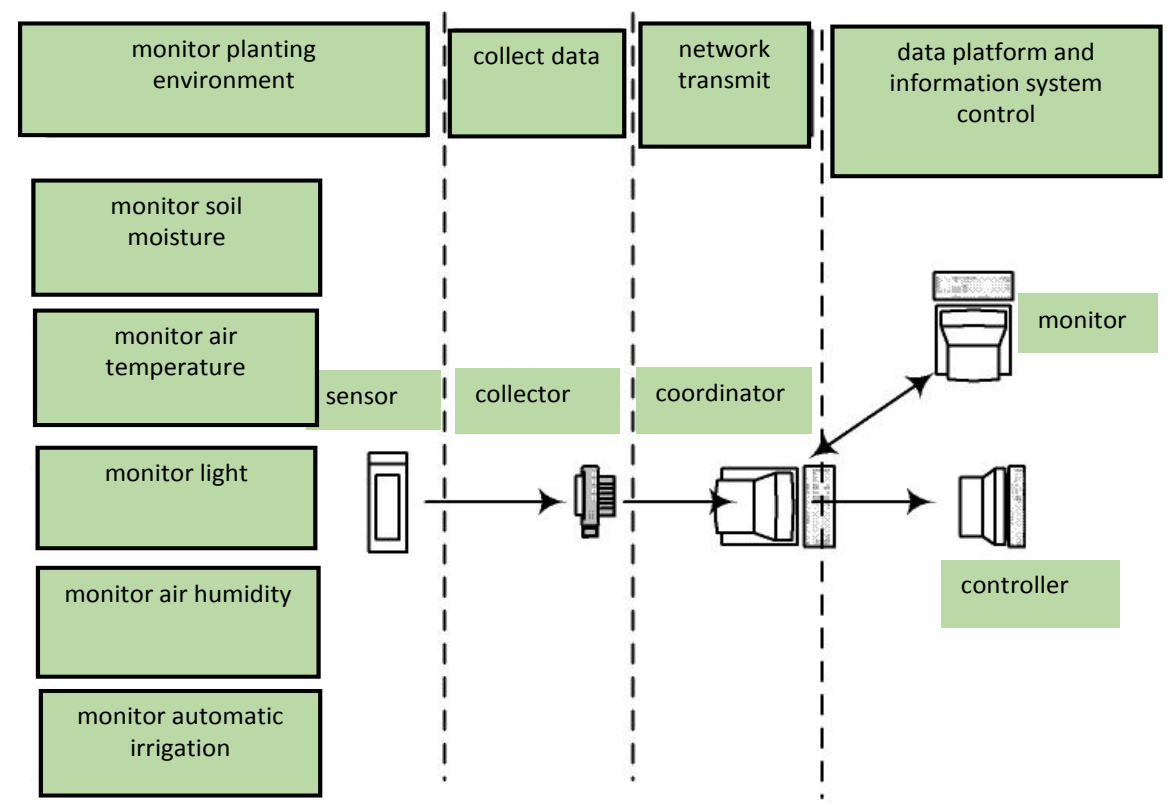

Figure 1. Diagram of intelligent agricultural planting system based on Internet of things technology.

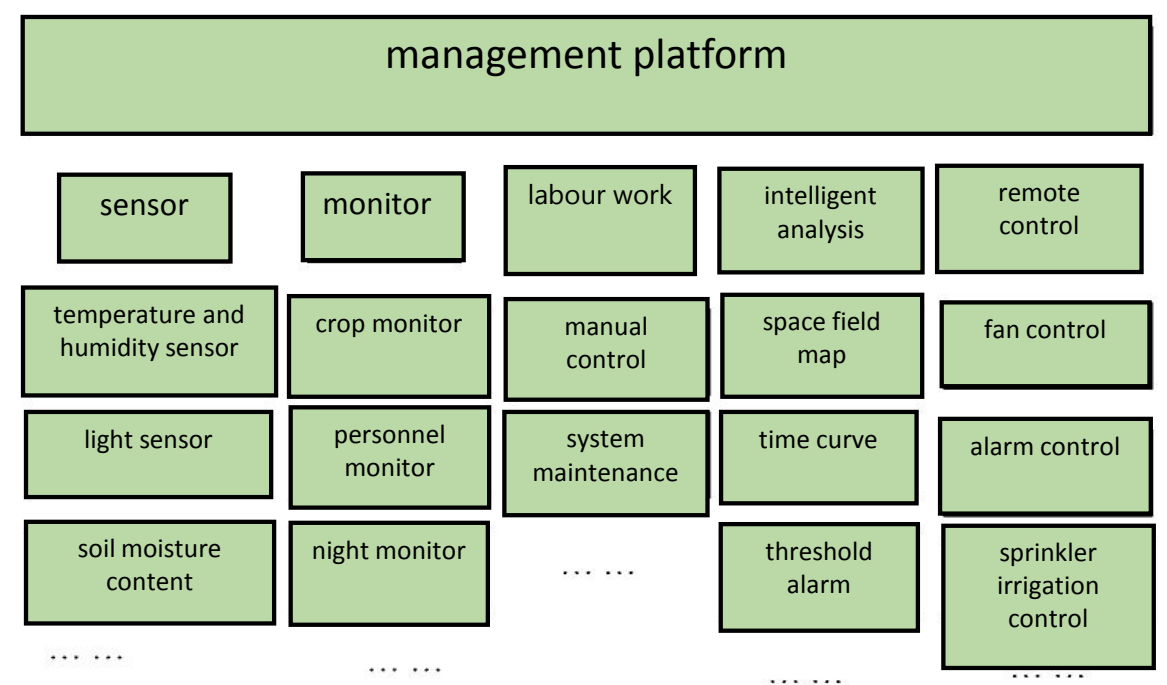

Figure 2. Diagram of intelligent agricultural planting system management platform based on Internet of things technology.

aims to improve the automation and intelligence level of modern agriculture, reduce resource share, improve the production efficiency and quality of agricultural products, and provides a certain basis for realizing automatic detection, automatic control, intelligent management and popularization of agricultural Internet of things in intelligent agriculture.

\section{Acknowledgements}

The project has been funded by the Sichuan science and technology and Intellectual Property Office of Nanchong, China (NC17SY2003). 


\section{References}

[1] Wang, Z., Li, C., Shao, H. and Sun, J. (2018) Eye Recognition with Mixed Convolutional and Residual Network (MiCoRe-Net). IEEE Access, 6, 17905-17912.

https://doi.org/10.1109/ACCESS.2018.2812208

[2] Rappaport, T.S., MacCartney, G.R., Samimi, M.K. and Sun, S. (2015) Wideband Millimeter-Wave Propagation Measurements and Channel Models for Future Wireless Communication System Design. IEEE Transactions on Communications, 63, 3029-3056. https://doi.org/10.1109/TCOMM.2015.2434384

[3] Wang, Z., Wang, D., Li, C., Xu, Y., Li, H. and Bao, Z. (2018) Deep Reinforcement Learning of Cell Movement in the Early Stage of C. elegans Embryogenesis. arXiv:1801.04600.

[4] Xu. G., Chen. L.P. and Zhang, R.R. (2010) Agricultural Internet of Things Application Research Based on Precision Irrigation. Computer Research and Development, 47, 333-337.

[5] Li, S. (2012) Research on Internet of Things and Smart Home Application. Modern Electronic Technology, 7, 120-123.

[6] Wang, B.Y. (2009) An Overview of the Internet of Things Research. Journal of Electronic Measurement and Instrumentation, 12, 17.

[7] Zhao, L. (2011) Discussion on the Application and Key Technical Requirements of Internet of Things in Agriculture. Telecom Science, 10, 70-73.

[8] Qi, L. (2011) Characteristics, Advantages and Applications of Internet of Things Agricultural Intelligent Measurement and Control System in Greenhouse. Anhui Agricultural Sciences, 30, 89-92. 